\title{
DISCRIMINAÇÃO SIMPLES E PAREAMENTO AO MODELO COM OBJETOS EM MACACOS-PREGO
}

INFANTES E ADULTOS.

DISCRIMINAÇÕES COM OBJETOS EM MACACOS-PREGO INFANTES E ADULTOS.

\author{
SIMPLE DISCRIMINATION AND MATCHING-TO-SAMPLE WITH OBJECTS IN INFANT AND \\ ADULT CAPUCHIN MONKEYS
}

DISCRIMINATIONS WITH OBJECTS IN INFANT AND ADULT CAPUCHIN MONKEYS

\author{
carlos B. A. Souza, abraĩo R. fonseca, liane J. S. Dahás \\ UnIVERsidAdE FEDERAL do PARÁ
}

\begin{abstract}
RESUMO
Este experimento procurou confirmar um resultado de aquisição de discriminação simples com mudanças sucessivas da função de estímulos (DSMS) e de matching-to-sample de identidade (IDMTS) com estímulos tridimensionais por macacos-prego (Sapajus, spp.) adultos e infantes. Dois macacos-prego infantes (M26 e M28) e dois adultos (M27 e M18) foram submetidos inicialmente a um treino de DSMS que alternou tentativas nas quais apenas um estímulo (o $\mathrm{S}+$ ) era apresentado com tentativas com dois ou mais estímulos apresentados simultaneamente. Foi feita uma redução gradual da probabilidade reforçamento nas tentativas com estímulo único, aproximando esse treino ao de IDMTS. O treino de IDMTS iniciou-se com dois modelos e duas comparações e prosseguiu até atingir quatro modelos e quatro comparações. Os sujeitos infantes aprenderam a tarefa de DSMS em 80 (M26) e 94 (M28) sessões, respectivamente, enquanto que M27 precisou de 103 sessões e M18 de 22 sessões. Os sujeitos M26, M27, M28 e M18 necessitaram, respectivamente, de 100, 36, 78 e 26 sessões para concluir o treino de IDMTS. Os desempenhos dos sujeitos foram comparados com aqueles de estudos com estímulos bidimensionais e discutidos em termos de suas histórias experimentais e do procedimento de DSMS usado neste estudo.
\end{abstract}

Palavras-chave: Discriminação simples; matching-to-sample de identidade; estímulos tridimensionais; macaco-prego.

\begin{abstract}
This study sought to confirm the acquisition of repeated shifts of simple discrimination (RSSD) and identity matching-tosample (IDMTS) using three-dimensional stimuli in adult capuchin monkeys (Sapajus, spp.). We also investigated the possibility that this acquisition can occur in infant capuchins. Two infants (M26 and M28) and two adults (M27 and M18) were given RSSD training, interspersing trials in which only one stimulus was presented $(\mathrm{S}+)$ with trials in which two or more stimuli were presented simultaneously. The reinforcement rate was then reduced in single stimulus trails so the procedure became similar to an IDMTS procedure. IDMTS training began with two samples and two comparisons, increasing to four samples and four comparisons. Subjects M26, M28, M27, and M18 completed the RSSD training in 80, 94, 103, and 22 sessions, respectively. In the IDMTS task, M26, M27, M28, and M18 needed 100, 36, 78, and 26 sessions to reach the criterion, respectively. The performance of the four subjects was compared to studies that used two-dimensional stimuli with regard to experimental history and the RSSD procedure used in this study.

Keywords: Simple discrimination; identity matching-to-sample; three-dimensional stimuli; capuchin monkey.
\end{abstract}

Trabalho realizado com apoio do CNPq na forma de Bolsa de Produtividade em Pesquisa para Carlos B. A. Souza e de Iniciação Científica para Abraão R. Fonseca e Liane J. S. Dahás.

Correspondência para Carlos B. A. Souza: carlosouz@gmail.com

Os autores agradecem a Edilson Pastana pelo trabalho de manutenção e alimentação dos animais da Escola Experimental de Primatas da UFPA. 
Discriminação simples e pareamento ao modelo com objetos em macacos-prego infantes e adultos

Responder a relações arbitrárias entre estímulos sonoros, visuais e táteis é um comportamento que parece ser de grande importância no ajuste de diferentes espécies ao seu ambiente (Deacon, 1997; Tomasello, \& Call, 1997). Esse tipo de comportamento, característico do que é denominado comportamento simbólico tem sido observado com diferentes níveis de funcionalidade em primatas humanos e não-humanos (Hixson, 1998; Horne, Lowe, \& Harris, 2007; Martinez, \& Matsuzawa, 2009a, 2009b; Savage-Rumbaugh et al., 1993).

Uma espécie de primata não-humano que tem sido utilizada em estudos sobre aquisição de repertórios simbólicos é o macaco-prego (Sapajus, spp.) (e.g., Barros, Galvão, \& Mcllvane, 2002; Barros, Souza, \& Costa, 2013; Galvão, Barros, Rocha, Mendonça, \& Goulart, 2002; Galvão et al., 2005; Goulart, Mendonça, Barros, Galvão, \& McIlvane, 2005; Souza, Ramos, Galvão, \& Barros, 2008). Estudos avaliaram, por exemplo, o comportamento de macacos-prego em situações experimentais ou naturais (Fragaszy, Izar, Visalberghi, Ottoni, \& Oliveira, 2004; Ottoni, \& Izar, 2008; Resende, \& Ottoni, 2002; Tavares, \& Tomaz, 2002) e observaram que esta espécie aprende por imitação, apresenta discriminação espacial e utiliza ferramentas. Tais repertórios os qualificam como um possível modelo não humano experimental para pesquisas relacionadas a aquisição de repertórios simbólicos (Barros, Galvão, \& Mcllvane, 2003; Mcllvane et al., 2011; Nagahama \& Souza, 2013).

Um conjunto de estudos investigando relações de equivalência (ver Sidman, 2000) em macacos-prego tem sugerido que repertórios simbólicos podem ser adquiridos de forma consistente seguindo-se um "currículo" no qual a realização de tarefas mais simples poderia facilitar a realização de outra mais complexa (Barros et al., 2003; Galvão et al., 2002). Galvão et al. (2002) relataram que seis de oito macacos-prego expostos ao procedimento tipo "currículo" desempenharam a tarefa de identidade generalizada com sucesso, tendo passado antes por treinos de discriminação simples, reversões repetidas de discriminação simples (RRDS), discriminações simples com mudanças sucessivas nas funções dos estímulos $(\text { DSMS })^{1}$ e matching-to-sample de identidade (IDMTS) (ver Galvão et al., 2005, para uma análise mais detalhada dos resultados destes sujeitos).

Barros et al. (2002) verificaram, em um conjunto de três experimentos com dois macacosprego, utilizando a mesma lógica de procedimento "currículo", que ambos os sujeitos apresentaram o responder generalizado por identidade após treinos de
RRDS e DSMS. Goulart et al. (2005) verificaram ainda que o uso do procedimento de comparação vazio (no qual um quadrado branco "substitui" ora o estímulo com função de S+ ora aquele[s] com função de S-; ver Wilkinson \& McIlvane, 1997) podia ajudar a avaliar o tipo de controle sobre as escolhas, se por seleção do $\mathrm{S}+$ e/ou por rejeição do S- no desempenho de RRDS, e que o treino nesta tarefa empregando este procedimento facilitou a aquisição de ambos os tipos de controle.

Deve-se ressaltar que, nos estudos mencionados anteriormente, os treinos e testes nas diferentes tarefas foram sempre realizados com estímulos bidimensionais. Porém, em seu ambiente natural o macaco-prego lida, em geral, com tarefas de discriminação visual de objetos tridimensionais (Fragaszy, Visalbergui, \& Fedigan, 2004), apresentando, como mencionado antes, uso de ferramentas para diferentes finalidades (Moura, \& Lee, 2005; Ottoni, \& Izar, 2008). Uma questão que se coloca, portanto, é se o uso de tarefas que permitissem aos macacos-prego manipular objetos poderia facilitar a aquisição de repertórios discriminativos em situações de discriminação simples e condicional.

Lima et al. (2007) avaliaram essa possibilidade submetendo dois macacos-prego a um conjunto de tarefas com estímulos tridimensionais (objetos) que incluía três etapas distintas. Inicialmente, aplicaram um treino de RRDS; após a aprendizagem nesta tarefa, seguiu-se um treino de IDMTS; e, por fim, um teste de identidade generalizada. Ambos os sujeitos tinham história experimental nestas mesmas tarefas com estímulos bidimensionais. Um dos sujeitos alcançou o critério de aprendizagem ( $90 \%$ de acertos) no treino de RRDS em 14 sessões e o outro em 17. No treino de IDMTS, um dos sujeitos levou 28 sessões e outro, 30 sessões para alcançar o critério $(90 \%$ de acertos). Os dois sujeitos mostraram evidência de aquisição de identidade generalizada somente quando os estímulos testados haviam passado previamente pelo procedimento de RRDS. Esse desempenho que não diferiu daquele observado na maioria dos sujeitos dos experimentos que utilizaram estímulos bidimensionais (mas ver dados obtidos com o sujeito M15, relatados em Galvão et al., 2005). Além disso, como apontaram Lima et al., uma diferença entre os dados do seu estudo "...e os dados de estudos anteriores com estímulos bidimensionais é que no presente estudo foi necessário realizar um número relativamente pequeno de sessões de reversões de discriminações simples e de pareamento ao modelo por identidade." (p.17)

Alguns estudos com outras espécies têm sugerido que o treino com estímulos tridimensionais pode facilitar a aprendizagem de IDMTS e emergência

\footnotetext{
RRDS é um procedimento no qual dois estímulos são apresentados ao sujeito (p. ex., A1+e A2-). Somente as respostas de escolha a um deles (A1+) são reforçadas. Quando o sujeito responde consistentemente a esse estímulo, as funções de S+ e S- são revertidas (A2+/A1-), passando a ser reforçadas respostas apenas ao outro estímulo. Quando o desempenho se torna preciso é realizada outra reversão, e assim por diante. Quando são utilizados três ou mais estímulos, o termo mais adequado para descrever a mudança da função dos estímulos é discriminações simples com mudanças sucessivas nas funções dos estímulos (DSMS).
} 
de identidade generalizada. Herman, Gory, Hovancik e Bradshaw (1989) investigaram a aquisição de IDMTS em um golfinho (Tursiops truncatus). Em um conjunto de quatro experimentos que avaliaram os efeitos (1) da apresentação como modelos de objetos acompanhados ou não de estímulos auditivos (nomes dos objetos em uma linguagem artificial); (2) de atrasos na apresentação das comparações; e (3) da apresentação de estímulos modelos estáticos ou em movimento, estes autores verificaram que o sujeito emparelhou por identidade 16 de 18 objetos na primeira tentativa na qual eles apareceram como modelos.

Pack, Herman e Roitblat (1991) verificaram que um leão-marinho (Zalophus californianus) aprendeu o repertório de IDMTS com dois objetos após um treino relativamente rápido (12 sessões, totalizando 269 tentativas - com 70 erros). Testes posteriores de IDMTS com pares de estímulos constituídos por (1) um objeto novo e cada um dos objetos usados no treino inicial, e (2) por objetos novos, mostraram um nível de acerto de aproximadamente $80 \%$. Também Kastak e Schusterman (1994- Experimento 2) obtiveram evidência de identidade generalizada com objetos em dois leões-marinhos depois de um treino de IDMTS com 15 pares de objetos.

No estudo que serviu de base para Lima et al. (2007), Oden, Thompson e Premack (1988) treinaram quatro chimpanzés (Pan troglodytes) infantes em uma tarefa de IDMTS com dois objetos. Após o critério de aprendizagem de 10 a 12 respostas corretas consecutivas para cada relação, os sujeitos foram expostos às sessões de testes com 12 novos estímulos (sem reforçamento diferencial, i.e., todas as respostas eram reforçadas). Observou-se que os sujeitos acertaram de $75 \%$ a $85 \%$ das tentativas de teste de identidade generalizada. Este estudo mostrou ainda um dado que tem sido sugerido nas pesquisas sobre aquisição de repertórios simbólicos com primatas: certa facilidade/superioridade de infantes na aquisição deste tipo de repertório (e.g., Savage-Rumbaugh et al., 1993; Sousa, Okamoto, \& Matsuzawa, 2003).

O presente trabalho procurou confirmar os dados do estudo de Lima et al. (2007) sobre a aquisição de discriminação simples (com reversões da função dos estímulos) e de IDMTS com estímulos tridimensionais (objetos) por macacos-prego. Investigou-se também a possibilidade da aquisição destes repertórios por macacos-prego infantes (com menos de dois anos de idade - Fragaszy et al., 2004).

\section{MÉTODO}

\section{Sujeitos}

Quatro macacos-prego (Sapajus, spp.) machos. Dois infantes, M26 com cerca de 1 ano de idade e M28, com pouco mais de 2 anos, ambos experimentalmente ingênuos. Um adulto-jovem, M27, de 4 anos, experimentalmente ingênuo e um adulto, M18, com pouco mais de 4 anos, com história de treino em discriminações simples e condicionais com estímulos bidimensionais. As condições de manutenção, manejo, alimentação e saúde, e os procedimentos experimentais foram aprovados pelo IBAMA e seguiram as normas da APA de condução ética no cuidado e uso de animais. Os procedimentos foram aprovados pelo Comitê de Ética em Pesquisa com Animais da Universidade Federal do Pará (CEPAE-UFPA: PS001/2005).

\section{Equipamentos}

Uma câmara experimental de $1 \mathrm{~m}^{2}$ com um lado fechado por material transparente e os restantes por uma tela de ferro. Em um dos lados com tela havia uma porta de $25 \times 40 \mathrm{~cm}$ pela qual o sujeito era introduzido e retirado da gaiola. Nesta porta havia quatro aberturas de $5 \times 7 \mathrm{~cm}$ para apresentação e seleção dos estímulos (objetos), que eram colocados à frente da porta em uma bandeja de aproximadamente $40 \times 40 \mathrm{~cm}$ divididos em nove quadrantes de mesma medida que serviam como posições para apresentação dos estímulos. Na parede adjacente à direita da porta, havia uma abertura de 10 x $10 \mathrm{~cm}$ para o sujeito colocar os objetos pegos por ele (ver Procedimento). Acoplado à câmara, havia dois dispensadores automáticos de pelotas (Med Associates - Inc) que liberavam pelotas de $190 \mathrm{mg}$ de alimento em um coletor localizado abaixo da abertura para a devolução dos objetos.

Um computador (Intel Pentium III) com o Programa TREL V2.1. (desenvolvido por José Iran Ataíde dos Santos) apresentava os estímulos na tela de um monitor, e o experimentador colocava na bandeja os objetos correspondentes. O programa controlava a sequência de tentativas e a posição para apresentação dos objetos, registrava as respostas do sujeito (via resposta do experimentador na tela sensível ao toque, ver Procedimento) e acionava o dispensador de pelota quando a resposta era correta.

\section{Estímulos}

Um triângulo azul (A1), um círculo roxo (A2), uma estrela rosa de cinco pontas (A3), uma elipse laranja (A4), e uma estrela verde de oito pontas (A5), todos de plástico, com cerca de $5 \times 5 \times 2 \mathrm{~cm}$. Estes estímulos foram utilizados nos treinos de discriminação simples e de IDMTS.

\section{Procedimento}

$\mathrm{Na}$ primeira fase foram modelados os comportamentos dos sujeitos de entrar na câmara experimental e de devolver (via abertura na parede da câmara) objetos entregues para eles. Depois disto, foi realizado o treino de discriminações simples com mudanças sucessivas nas funções dos estímulos (DSMS) com estímulos tridimensionais. Como 
este treino tinha por objetivo facilitar a aquisição do repertório de IDMTS, e que estudos anteriores mostravam que geralmente os sujeitos levavam diversas sessões para passar de um treino para o outro (e.g., Brandão, 2001; Goulart, Galvão, \& Barros, 2003; Lavratti, 2002), foi utilizado neste estudo um novo procedimento para treinar discriminação simples. Este treino alternava tentativas nas quais um único estímulo (o $\mathrm{S}+$ ) era apresentado (tentativas com estímulo único) com tentativas nas quais dois ou mais estímulos eram apresentados (tentativas de escolha), sendo um deles o $\mathrm{S}+$ (o mesmo da tentativa com estímulo único imediatamente anterior, ver Rico, 2006). Como esta apresentação de estímulos se assemelha àquela da situação de IDMTS (com as diferenças que responder ao estímulo único era inicialmente reforçado e que havia um intervalo maior entre a apresentação do estímulo único e, posteriormente, das escolhas), trabalhou-se com a hipótese de que ela pudesse facilitar a passagem para o treino de IDMTS. Em ambos os tipos de treino, realizou-se uma sessão por dia, de segunda a sexta-feira.

\section{Treino de discriminações simples com mudanças sucessivas nas funções dos estímulos (DSMS).}

Cada tentativa iniciava com um estímulo com função de $\mathrm{S}+$ sendo colocado sozinho na bandeja (tentativa com estímulo único), podendo ocupar uma de nove possíveis posições em uma matriz de 3 x 3 na bandeja (ver Barros et al., 2002). A configuração era mostrada na tela do computador exclusivamente para o experimentador, servindo como gabarito para construir a tentativa com os objetos correspondentes na bandeja. A resposta de pegar e devolver os objetos pela abertura na câmara experimental era consequenciada pela liberação de uma pelota de alimento (o experimentador tocava o estímulo correspondente na tela sensível ao toque do computador, o que produzia a liberação da pelota). Imediatamente após essa resposta, o mesmo objeto era recolocado na bandeja, novamente como $\mathrm{S}+$, mas agora simultaneamente com outro objeto com função de S(tentativa de escolha); cada objeto podia ocupar uma de nove possíveis posições na bandeja. Após a seleção e devolução do S+ ou do S-, uma nova configuração de tentativa com estímulo único era mostrada na tela do computador, e o experimentador construía a nova tentativa na bandeja, proporcionando um Intervalo Entre Tentativas (IET) de aproximadamente $6 \mathrm{~s}$, e uma nova tentativa era iniciada.

As primeiras sessões de treino de DSMS apresentavam 20 tentativas com o par de estímulos A1+ e A2-, sendo o critério de aprendizagem estabelecido em $90 \%$ de acertos. Após a $13^{\mathrm{a}}$ sessão, com a finalidade de possibilitar um número maior de mudanças de discriminações, o critério de aprendizagem foi alterado para oito respostas corretas consecutivas nas tentativas de escolha. As sessões terminavam quando o critério era atingido ou após 40 tentativas (ver Tabela 1).

Quando o critério de aprendizagem era alcançado para o primeiro par de estímulos, suas funções eram revertidas (A2+, A1-). Depois que o desempenho dos sujeitos alcançava novamente o critério, era acrescentado às tentativas de escolha um novo estímulo, com função negativa (A2+, A1-, A3-). Assim que o desempenho do sujeito atingia o critério com estes três estímulos, o estímulo A3 passava a ter função de S+(A3+, A1-, A2-). Quando se alcançava o critério nesta condição, o estímulo A1 era novamente apresentado como $\mathrm{S}+(\mathrm{A} 1+, \mathrm{A} 2-, \mathrm{A} 3-)$. Após a obtenção do critério nesta condição, um quarto estímulo era apresentado nas tentativas de escolha, mantendo-se o estímulo anterior como $\mathrm{S}+(\mathrm{A} 1+, \mathrm{A} 2-$, A3-, A4-). Após o critério nesta condição, a função positiva era estabelecida para o novo estímulo e, em seguida, mediante alcance do critério de precisão, para os outros dois estímulos (A3+, A2-, A1-, A4- e A2+, A1-, A3-, A4-; ver Tabela 1).

Após esta etapa do treino de DSMS, programouse uma sequência de sessões com reduções na probabilidade de reforçamento nas tentativas com estímulo único. Após a sexta mudança de função dos estímulos, a probabilidade foi reduzida para $50 \%$, e após o critério de aprendizagem, ainda nesta condição (A2+, A1-, A3-, A4-), foram reforçadas somente as cinco primeiras respostas de escolha do estímulo único de cada sessão. Após o critério nesta condição, foi feita uma nova mudança na função dos estímulos (A3+, A1-, A2-, A4-), mantendo-se reforçamento programado nas cinco primeiras tentativas com estímulo único. Após o critério, mas ainda na mesma condição, foram realizadas sessões com reforçamento programado para as três primeiras tentativas com estímulo único. Após o critério nesta condição, foi feita uma nova mudança na função dos estímulos (A4+, A1-, A2-, A3-), mantendo-se o reforçamento das escolhas nas três primeiras tentativas com estímulo único. Após o critério, mas ainda na mesma condição, foram realizadas sessões sem reforçamento programado nas tentativas com estímulo único. Por fim, após o critério nesta última condição, foi feita uma nova mudança na função dos estímulos, mantendo-se as tentativas com estímulo único sem reforçamento (A1+, A2-, A3-, A4-) (ver Tabela 1). Esperava-se que estas sessões sem reforço para as respostas nas tentativas com estímulo único, devido à semelhança de configuração com o treino de IDMTS, facilitassem a transição para este treino. 


\begin{tabular}{|c|c|c|c|c|c|c|c|}
\hline \multicolumn{5}{|c|}{ TENTATIVAS } & \multirow{2}{*}{\multicolumn{2}{|c|}{ Reforco nas }} & \multirow{3}{*}{$\begin{array}{l}\text { Tentativas } \\
\text { por sessão }\end{array}$} \\
\hline E. & & Esc & & & & & \\
\hline Único & S+ & & S- & & E. único & Critério & \\
\hline A1 & $\mathrm{A} 1$ & A2 & & & $100 \%$ & $90 \%$ & 20 \\
\hline $\mathrm{A} 2$ & $\mathrm{~A} 2$ & A1 & - & - & $100 \%$ & $90 \%$ & 20 \\
\hline $\mathrm{A} 2$ & $\mathrm{~A} 2$ & A1 & A3 & - & $100 \%$ & $90 \%$ & 20 \\
\hline $\mathrm{A} 3$ & $\mathrm{~A} 3$ & A1 & $\mathrm{A} 2$ & - & $100 \%$ & $8 c c$ & 40 \\
\hline $\mathrm{A} 1$ & $\mathrm{~A} 1$ & $\mathrm{~A} 2$ & A3 & - & $100 \%$ & $8 \mathrm{cc}$ & 40 \\
\hline $\mathrm{A} 1$ & A1 & $\mathrm{A} 2$ & A3 & A4 & $100 \%$ & $8 \mathrm{cc}$ & 40 \\
\hline A4 & A4 & A2 & A3 & $\mathrm{A} 1$ & $100 \%$ & $8 c c$ & 40 \\
\hline $\mathrm{A} 3$ & A3 & A2 & $\mathrm{A} 1$ & $\mathrm{~A} 4$ & $100 \%$ & $8 c c$ & 40 \\
\hline $\mathrm{A} 2$ & $\mathrm{~A} 2$ & A1 & A3 & A4 & $100 \%$ & $8 c c$ & 40 \\
\hline $\mathrm{A} 2$ & $\mathrm{~A} 2$ & A1 & A3 & A4 & $50 \% / 5 \mathrm{pt}$ & $8 \mathrm{cc}$ & 40 \\
\hline $\mathrm{A} 3$ & A3 & $\mathrm{A} 1$ & $\mathrm{~A} 2$ & A4 & $5 \mathrm{pt} / 3 \mathrm{pt}$ & $8 c c$ & 40 \\
\hline A4 & $\mathrm{A} 4$ & A1 & $\mathrm{A} 2$ & $\mathrm{~A} 3$ & 3pt. / 0 & $8 c c$ & 40 \\
\hline A1 & $\mathrm{A} 1$ & $\mathrm{~A} 2$ & A3 & A4 & 0 & $8 \mathrm{cc}$ & 40 \\
\hline
\end{tabular}

E. único= tentativa com estímulo único. $\mathrm{S}+=$ estímulo com função positiva. $\mathrm{S}-=$ estímulos com função negativa. $8 \mathrm{cc}=$ oito respostas corretas consecutivas em tentativas de escolha. $5 \mathrm{pt} \mathrm{e} 3 \mathrm{pt}=$ Cinco e três primeiras tentativas com estímulo único da sessão. Ver texto para explicação mais detalhada.

\section{Treino de matching-to-sample de identidade (IDMTS).}

Cada tentativa iniciava com um objeto (estímulo modelo) sendo colocado pelo experimentador em uma das nove possíveis posições da bandeja. A resposta de pegá-lo e devolvê-lo pela abertura na câmara experimental tinha como consequência a apresentação de um objeto idêntico ao modelo (S+) e de outro(s) estímulo(s) de comparação (S-) em duas ou mais das nove posições da bandeja (as configurações dos estímulos em cada tentativa eram mostradas na tela do computador para guiar a conduta do experimentador na montagem das tentativas, como no treino de DSMS). A resposta de seleção e devolução do S+ era consequenciada pela liberação de uma pelota de alimento (via resposta do experimentador na tela sensível ao toque) e produzia um IET de aproximadamente $6 \mathrm{~s}$ (produzido também pela resposta de seleção e devolução de um S-), depois do qual se iniciava uma nova tentativa, que poderia apresentar o mesmo estímulo modelo ou outro estímulo do conjunto treinado (ver a seguir). As sessões eram de 40 tentativas.

Em uma primeira fase do treino de IDMTS, era apresentado o mesmo modelo nas 20 primeiras tentativas, e outro estímulo como modelo nas 20 tentativas restantes, tendo somente os dois estímulos comparações (o critério de aprendizagem era de $80 \%$ de acertos com cada estímulo como modelo - critério mantido no restante do treino de IDMTS). Depois de atingido o critério com os dois primeiros estímulos, o procedimento era repetido com os outros dois. Atingido o critério com estes dois, passou-se para a condição na qual eram realizadas sessões com quatro estímulos, aparecendo como modelo, mas somente dois como comparações. Nesta fase, primeiro foram realizadas sessões com dez tentativas consecutivas para cada modelo. Após o critério, passou-se para o treino de oito blocos de cinco tentativas consecutivas de mesmo modelo, e depois para blocos de cinco (inicialmente) e de duas tentativas consecutivas com o mesmo modelo. Por fim, nesta condição com quatro modelos e duas comparações, foram realizadas sessões onde a sequênwcia das tentativas era totalmente randomizada após a décima tentativa. Após o critério nesta condição, foram programadas sessões totalmente randomizadas com quatro estímulos como modelos, tendo-se inicialmente três comparações, e após o critério, sessões com quatro comparações (ver Tabela 2). 
Número de comparações, modelos apresentados e blocos de tentativas utilizadas durante as etapas do treino de IDMTS

\begin{tabular}{|c|c|c|c|}
\hline $\begin{array}{l}\text { Número de } \\
\text { Comparações }\end{array}$ & Modelos & Tentativas & Agrupamento de Tentativas \\
\hline 2 & $\mathrm{~A} 1, \mathrm{~A} 2$ & & 2 blocos de 20 tentativas consecutivas \\
\hline 2 & $\mathrm{~A} 3, \mathrm{~A} 4$ & & 2 blocos de 20 tentativas consecutivas \\
\hline 2 & $\mathrm{~A} 1, \mathrm{~A} 2, \mathrm{~A} 3, \mathrm{~A} 4$ & & 4 blocos de 10 tentativas consecutivas \\
\hline 2 & $\mathrm{~A} 1, \mathrm{~A} 2, \mathrm{~A} 3, \mathrm{~A} 4$ & 40 & 8 blocos de 5 tentativas consecutivas \\
\hline 2 & $\mathrm{~A} 1, \mathrm{~A} 2, \mathrm{~A} 3, \mathrm{~A} 4$ & & Blocos de 5 e 2 tentativas consecutivas \\
\hline 2 & $\mathrm{~A} 1, \mathrm{~A} 2, \mathrm{~A} 3, \mathrm{~A} 4$ & & Tentativas randomizadas após a $10^{\mathrm{a}}$ tentativa \\
\hline 3 & $\mathrm{~A} 1, \mathrm{~A} 2, \mathrm{~A} 3, \mathrm{~A} 4$ & & Tentativas randomizadas \\
\hline 4 & $\mathrm{~A} 1, \mathrm{~A} 2, \mathrm{~A} 3, \mathrm{~A} 4$ & & Tentativas randomizadas \\
\hline
\end{tabular}

Resultados e Discussão

A Tabela 3 mostra o desempenho dos sujeitos no treino de DSMS. O sujeito infante M26 precisou de 80 sessões para concluir este treino. Ele atingiu o critério de aprendizagem após a primeira mudança na função dos estímulos em oito sessões, e precisou de 40 sessões para alcançar os critérios de aprendizagem nas cinco demais mudanças na função dos estímulos durante o treino. Com o início da redução de reforçamento nas tentativas com estímulo único, M26 necessitou de 18 sessões para atingir o critério nas próximas duas mudanças $\left(7^{\mathrm{a}}\right.$ e $\left.8^{\mathrm{a}}\right)$ e alcançou o critério de aprendizagem na última mudança, já sem reforçamento nas tentativas com estímulo único, em 10 sessões.

O sujeito infante M28 concluiu o treino de DSMS em 94 sessões. Para atingir o critério após a primeira mudança na função dos estímulos o sujeito precisou de 21 sessões. Além disso, foram necessárias 48 sessões para alcançar tais critérios nas cinco mudanças na função dos estímulos no treino. Com o início da redução de reforçamento nas tentativas com estímulo único, M28 precisou de quatro sessões para atingir o critério nas $7^{\mathrm{a}}$ e $8^{\mathrm{a}}$ mudanças, e alcançou o critério de aprendizagem na última mudança também em 10 sessões.

$\mathrm{O}$ sujeito M27, adulto e ingênuo experimentalmente, precisou de 103 sessões para concluir o treino de DSMS. Ele atingiu o critério de aprendizagem após a primeira mudança na função dos estímulos em 18 sessões, e precisou de 53 sessões para alcançar as cinco mudanças na função dos estímulos no treino. Com a redução de reforçamento nas tentativas com estímulo único, M27 necessitou de 12 sessões para atingir o critério nas próximas duas mudanças $\left(7^{\mathrm{a}}\right.$ e $\left.8^{\mathrm{a}}\right)$, alcançando o critério de aprendizagem na última mudança em nove sessões.
O sujeito M18, adulto e com história de treino em discriminações simples e condicionais com estímulos bidimensionais, finalizou o treino de DSMS em 22 sessões. Ele levou três sessões para atingir o critério após a primeira mudança na função dos estímulos, e precisou de 12 sessões para alcançar as cinco mudanças na função dos estímulos no treino com reforçamento nas tentativas com estímulo único. Com o início da redução de reforçamento nas tentativas com estímulo único, M18 precisou de duas sessões para atingir o critério nas $7^{\mathrm{a}}$ e $8^{\mathrm{a}}$ mudanças, e alcançou o critério de aprendizagem na última mudança em apenas uma sessão.

A retirada total do reforçamento nas tentativas com estímulo único prejudicou o desempenho de M26, M27 e M28 (como pode ser visto pela reversão na tendência de diminuição do número de sessões para alcançar o critério de aprendizagem registrado nas duas últimas mudanças nas funções dos estímulos). Essa queda no desempenho não foi observada nos sujeitos do experimento de Souza, Dahás e Barros (2011), o qual demonstrou que macacos-prego infantes aprendem DSMS e IDMTS com estímulos bidimensionais. No referido estudo, os dois sujeitos precisaram de um total de 59 e 52 sessões para terminar o treino de DSMS. Essa diferença pode ter ocorrido em função de um efeito diferencial da redução do reforçamento sobre o desempenho em tarefas com estímulos bi e tridimensionais. Esta possibilidade deve ser avaliada em estudos futuros. 
Configuração das tentativas, momento de ocorrência de mudanças de função e número de sessões no treino de DSMS para cada sujeito

\begin{tabular}{|c|c|c|c|c|c|c|c|c|}
\hline \multicolumn{4}{|c|}{ Estímulos } & \multirow{2}{*}{$\begin{array}{l}\text { Mudanças } \\
\text { de Função }\end{array}$} & \multicolumn{4}{|c|}{ Sessões/sujeitos } \\
\hline $\mathbf{S}+$ & & S- & & & M18 & M26 & M27 & M28 \\
\hline $\mathrm{A} 1$ & $\mathrm{~A} 2$ & & & & 1 & 2 & 9 & 9 \\
\hline A2 & $\mathrm{A} 1$ & - & - & $1^{\mathrm{a}}$ & 3 & 8 & 18 & 21 \\
\hline $\mathrm{A} 2$ & $\mathrm{~A} 1$ & A3 & - & & 1 & 3 & 12 & 2 \\
\hline A3 & $\mathrm{A} 1$ & A 2 & - & $2^{\mathrm{a}}$ & 1 & 10 & 10 & 16 \\
\hline A1 & $\mathrm{A} 2$ & A3 & - & $3^{\mathrm{a}}$ & 3 & 5 & 10 & 17 \\
\hline A1 & $\mathrm{A} 2$ & A3 & A4 & & 1 & 7 & 3 & 1 \\
\hline A4 & $\mathrm{A} 2$ & A3 & A1 & $4^{\mathrm{a}}$ & 3 & 4 & 6 & 2 \\
\hline A3 & $\mathrm{A} 2$ & A1 & A4 & $5^{\mathrm{a}}$ & 2 & 8 & 7 & 7 \\
\hline A2 & $\mathrm{A} 1$ & A3 & A4 & $6^{\mathrm{a}}$ & 1 & 3 & 5 & 3 \\
\hline $\mathrm{A} 2$ & $\mathrm{~A} 1$ & A3 & A4 & & 1 & 2 & 2 & 2 \\
\hline A3 & $\mathrm{A} 1$ & A 2 & A4 & $7^{\mathrm{a}}$ & 1 & 5 & 6 & 3 \\
\hline A4 & $\mathrm{A} 1$ & A2 & A3 & $8^{\mathrm{a}}$ & 1 & 13 & 6 & 1 \\
\hline \multirow[t]{2}{*}{ A1 } & $\mathrm{A} 2$ & A3 & A4 & $9^{a}$ & 1 & 10 & 9 & 10 \\
\hline & & & & TOTAL & 22 & 80 & 103 & 94 \\
\hline
\end{tabular}

A Tabela 4 mostra o desempenho dos sujeitos na tarefa de IDMTS. O sujeito infante M26 levou 100 sessões para finalizar esse treino. Na fase inicial, com dois modelos e duas comparações em dois blocos de 20 tentativas, M26 levou 16 sessões para alcançar o critério de aprendizagem. $\mathrm{Na}$ fase com quatro modelos e duas comparações e redução gradual no tamanho dos blocos de tentativas, ele necessitou de 13 sessões para alcançar o critério. Na fase com quatro modelos e três comparações, com tentativas randomizadas, M26 permaneceu 34 sessões sem alcançar o critério, com desempenho instável nos estímulos A1 e, principalmente, no A3. A partir da $35^{\mathrm{a}}$ sessão, o estímulo A3 foi substituído pelo estímulo A5, e começou a ser realizado o procedimento de correção (no qual uma tentativa era apresentada repetidamente em todas as suas propriedades até que a resposta correta fosse emitida). Após estas alterações M26 alcançou o critério em 23 sessões. Por último, na condição com quatro modelos e quatro comparações, com tentativas randomizadas, ele necessitou de 14 sessões para alcançar o critério de aprendizagem.

O sujeito infante M28 necessitou 78 sessões para concluir o treino de IDMTS. Na fase com dois modelos e duas comparações, ele alcançou o critério de aprendizagem também em 16 sessões. Na fase com quatro modelos e duas comparações, ele necessitou de 16 sessões para alcançar o critério de aprendizagem. Por fim, nas fases com tentativas randomizadas, com quatro modelos e três comparações M28 alcançou o critério de aprendizagem em 27 sessões, e com quatro modelos e quatro comparações em 19 sessões.

O sujeito adulto ingênuo M27 levou 36 sessões para concluir o treino de IDMTS. Na fase com dois modelos e duas comparações, ele alcançou o critério de aprendizagem em 11 sessões. $\mathrm{Na}$ fase com quatro modelos e duas comparações, ele precisou de 15 sessões para alcançar o critério. Na fase com quatro modelos e três comparações (com tentativas randomizadas), M27 alcançou o critério em sete sessões. Por último, na condição com quatro modelos e quatro comparações, com tentativas randomizadas, o sujeito alcançou o critério em três sessões.

O sujeito adulto M18 levou 26 sessões para concluir o treino de IDMTS. Ele alcançou o critério de aprendizagem em duas sessões na fase com dois modelos e duas comparações. Na fase com quatro modelos e duas comparações, ele necessitou de 12 sessões para alcançar o critério. Na fase com quatro modelos e três comparações, M18 alcançou o critério em nove sessões, e na fase com quatro modelos e quatro comparações em três sessões.

Estes resultados dão suporte aos dados de Lima et al. (2007), sugerindo que macacos-prego podem aprender repertórios de DSMS e IDMTS com estímulos tridimensionais (objetos). Além disso, demonstram pela primeira vez a aquisição destes repertórios com este tipo de estímulo por macacosprego infantes.

Deve-se ressaltar que, diferentemente do estudo de Lima et al. (2007), no presente estudo foi utilizado o procedimento de tentativas com estímulo único no treino de DSMS, aproximando o procedimento ao de IDMTS. Isso parece ter facilitado a aquisição do repertório por parte dos sujeitos. Considerando-se que no estudo de Lima et al. (2007) os treinos de RSDS e IDMTS foram realizados apenas com dois estímulos, o número de sessões necessárias para o critério em condições equivalentes no presente estudo (i.e., os treinos iniciais com dois estímulos discriminativos em DSMS e com duas comparações em IDMTS) foram bem menores. 
para cada sujeito

\begin{tabular}{cccccc}
\hline $\mathbf{N}^{\mathbf{0}}$ de & & \multicolumn{4}{c}{ Sessões/Sujeitos } \\
\cline { 3 - 6 } Comparações & Modelos & M18 & M26 & M27 & M28 \\
\hline 2 & A1, A2 & 1 & 9 & 9 & 6 \\
2 & A3, A4 & 1 & 7 & 2 & 10 \\
2 & A1, A2, A3, A4 & 1 & 2 & 6 & 5 \\
2 & A1, A2, A3, A4 & 2 & 6 & 1 & 2 \\
2 & A1, A2, A3, A4 & 4 & 4 & 7 & 6 \\
3 & A1, A2, A3, A4 & 5 & 1 & 1 & 3 \\
4 & A1, A2, A3/A5 A4 & 9 & $57^{*}$ & 7 & 27 \\
& A1, A2, A5 A4 & 3 & 14 & 3 & 19 \\
\hline
\end{tabular}

*Para o sujeito M26, após a $34^{\text {a }}$ sessão nesta fase, ocorreu a substituição do estímulo A3 pelo estímulo A5 e a adoção do procedimento de correção (ver texto para detalhes).

Os desempenhos de M26, M28 (infantes ingênuos experimentalmente) e M27 (adulto ingênuo

experimentalmente) foram bem diferentes de M18 (adulto com história de treino em discriminações simples e condicionais com estímulos bidimensionais). M18 finalizou o treino em 48 sessões (22 de DSMS e 26 de IDMTS), enquanto M26 levou 180 sessões (80 de DSMS e 100 de IDMTS), M27 139 (103 de DSMS e 36 de IDMTS) e M28 172 (94 de DSMS e 78 de IDMTS). Se fossem considerados somente os dados das sessões de M26 após a substituição do estímulo A3 por A5, ele completaria o treino de IDMTS em 66 sessões. Isto ainda corresponde a mais que o dobro do que M18 necessitou. Esta diferença pode ser devida ao treino anterior de M18 nas mesmas tarefas, porém com estímulos bidimensionais (Rico, 2006), repertório que M26, M27 e M28 não tinham possivelmente sendo responsável pelo maior número de sessões destes.

Os resultados dos sujeitos infantes (M26 e M28) e do jovem-adulto (M27) deste estudo, quando comparados aos de Souza et al. (2011), sugerem uma maior facilidade na aquisição do repertório de DSMS com estímulos bidimensionais. No estudo de Souza et al., dois macacos-prego infantes levaram menos de 60 sessões para concluir o treino de DSMS, enquanto que o sujeitos infantes e o jovem-adulto do presente estudo precisaram, conforme já mencionado, de 80 sessões (M26), 103 sessões (M27) e 94 (M28). Esta diferença entre a aprendizagem com estímulos bidimensionais e tridimensionais pode ser função de uma maior complexidade discriminativa na tarefa com objetos, caracterizada em termos da dimensão discriminativa 'profundidade' presente em estímulos tridimensionais. Este dimensão implica propriedades discriminativas ao lidar com objetos (p. ex., variações de relevo e/ou volumétricas) que não estão presentes em tarefas com estímulos bidimensionais.

Os resultados deste estudo, tomados em conjunto com os de Lima et al. (2007), Souza et al. (2011) e Rico (2006), sugerem que: (1) para macacos-prego infantes, o repertório de DSMS parece ser adquirido mais facilmente com estímulos bidimensionais; (2) a exposição anterior ao procedimento com estímulos bidimensionais facilita a aquisição destes repertórios com objetos; e (3) o procedimento de tentativas com estímulo único durante o treino de DSMS parece facilitar a aquisição do repertório de IDMTS. Estudos futuros devem procurar analisar os efeitos específicos de cada uma destas variáveis. 


\section{REFERÊNCIAS}

Barros, R. S., Souza, C. B. A., \& Costa, T. D. (2013). Functional class formation in the context of a foraging task in capuchin monkeys. Journal of the Experimental Analysis of Behavior, 100, 79 87.

Barros, R. S., Galvão, O. F., \& McIlvane, W. J. (2002). Generalized identity matching to sample in Cebus apella. The Psychological Record, 52, 441 460.

Barros, R. S., Galvão, O. F., \& McIlvane, W. J. (2003). The search for relational learning capacity in Cebus apella. A programmed "educational" approach. In: S. Soraci Jr. \& K. Murata Soraci (Eds.), Visual information processing (pp. 223 245). Westport: Praeger Publishers.

Brandão, S. (2001). Programa de ensino de escolha de acordo com o modelo por identidade generalizada a partir de discriminaçôes simples com Cebus apella Dissertação de Mestrado. Universidade Federal do Pará, Belém do Pará.

Deacon, T. W. (1997). The symbolic species: The co evolution of language and the brain. New York: Norton.

Fragaszy, D. M., Izar, P., Visalberghi, E., Ottoni, E. B., \& Oliveira, M. G. (2004). Wild capuchin monkeys (Cebus libidinosus) use anvils and stone pounding tools. American Journal of Primatology, 64, 359 366.

Fragaszy, D. M., Visalberghi, E., \& Fedigan, L. (2004). The complete capuchin. Cambridge: Cambridge University Press.

Galvão, O. F., Barros, R. S., Rocha, A. C., Mendonça, M., \& Goulart, P. R. K. (2002). Escola experimental de primatas. Estudos de Psicologia, 7, 361 370.

Galvão, O. F., Barros, R. S., Santos, J. R., Brino, A. L. F., Brandão, S., Lavratti, C. M., Dube, W. V., \& McIlvane, W. J. (2005). Extent and limits of the matching concept in Cebus apella. A matter of experimental control? The Psychological Record, 55, 219 232.

Goulart, P. R. K., Galvão, O. F., \& Barros, R. S. B. (2003). Busca de formação de classes de estímulos via procedimento de reversões repetidas de discriminação simples combinadas em macacos prego (Cebus apella). Interação em Psicologia, 7, 109 120.

Goulart, P. R. K., Mendonça, M. B., Barros, R. S., Galvão, O. F., \& McIlvane, W. J. (2005). A note on select $\sim$ and reject $\sim$ controlling relations in the simple discrimination of capuchin monkeys (Cebus apella). Behavioural Processes, 69, 295 302.

Herman, L. M., Gory, J. D., Hovancik, J. R., \& Bradshaw, G. L. (1989). Generalization of visual matching by a bottlenosed dolphin (Tursiops truncatus). Evidence for invariance of cognitive performance with visual and auditory materials. Animal Behavior Processes, 15, 124 136.

Hixson, M. D. (1998). Ape language research: A review and behavioral perspective. The Analysis of Verbal Behavior, 15,17 39.

Horne, P. J., Lowe, C. F., \& Harris, F. D. A. (2007). Naming and categorization in young children: V. Manual sign training. Journal of the Experimental Analysis of Behavior, 87, 367 381.
Kastak, D., \& Schusterman, R. J. (1994). Transfer of visual identity matching to sample in two California sea lions (Zalophus californianus). Animal Learning $\&$ Behavior, 22, 427 435.

Lavratti, C. M. (2002). Condições suficientes (e talvez necessárias) para a emergência de pareamento ao modelo por identidade generalizada em Cebus apella. Dissertação de Mestrado. Universidade Federal do Pará, Belém do Pará.

Lima, M. E. A. C., Barros, R. S., Dahás, L. J. S., Cruz, A. P. C., Bezerra, D. S., \& Galvão, O. F. (2007). Discriminação simples e pareamento ao modelo por identidade com estímulos tridimensionais em macacos prego (Cebus apella). Acta Comportamentalia, 15, 5 20.

Martinez, L., \& Matsuzawa, T. (2009a). Auditory visual intermodal matching based on individual recognition in a chimpanzee (Pan troglodytes). Animal Cognition, 12, S71 S85.

Martinez, L., \& Matsuzawa, T. (2009b). Effect of species specificity in auditory visual intermodal matching in a chimpanzee (Pan troglodytes) and humans. Behavioural Processes, 82, 160 163.

McIlvane, W. J., Dube, W. V., Serna, R. W., Lionello DeNolf, K. M., Barros, R. S., \& Galvão, O. F. (2011). Some current dimensions of Translational Behavior Analysis: From laboratory research to intervention for persons with autism spectrum disorders. Em E. A. Mayville \& J. A. Mulick (Orgs.), Behavioral foundations of effective autism treatment (pp.155 181). Cornwall on Hudson, NY: Sloan Publishing.

Moura, A. C. A., \& Lee, P. C. (2005). Capuchin stone tool use in caatinga dry forest. Science, 306, 1909.

Nagahama, M. M., \& Souza, C. B. A. (no prelo). Escola Experimental de Primatas: Análise da coerência entre pressupostos e práticas empíricas. Revista Brasileira de Terapia Comportamental e Cognitiva. 2013.

Oden, D. L., Tompson, R. K. R., \& Premack, D. (1988). Spontaneous transfer of matching by infant chimpanzees (Pan troglodytes). Journal of Experimental Psychology: Animal Behavior Processes, 14, $140 \sim 145$.

Ottoni, E. B., \& Izar, P. (2008). Capuchin monkey tool use: Overview and implications. Evolutionary Anthropology, 17, 171 178.

Pack, A. A., Herman, L. M., \& Roitblat, H. L. (1991). Generalization of a visual matching and delayed matching by a California sea lion (Zalophus californianus). Animal Learning \& Behavior, 19,17 48.

Resende, B., \& Ottoni, E. (2002). Brincadeira e aprendizagem do uso de ferramentas em macacos prego (Cebus apella). Estudos de Psicologia 7, 173 180.

Rico, V. V. (2006). Persistência comportamental e topografía de controle de estímulos coerente em treino de discriminação simples e escolha condicional por identidade ao modelo com quatro escolhas em macaco prego (Cebus apella) Dissertação de Mestrado. Universidade Federal do Pará, Belém do Pará. 
Savage Rumbaugh, E., Murphy, J., Sevcik, R., Brakke, K., Williams, S., \& Rumbaugh, D. (1993). Language comprehension in ape and child. Monographs of the Society for Research in Child Development, 58, 1 256.

Sidman, M. (2000). Equivalence relation and the reinforcement contingency. Journal of the Experimental Analysis of Behavior, 74, 127 146.

Sousa, C., Okamoto, S., \& Matsuzawa, T. (2003). Behavioural development in a matching -to sample task and token use by an infant chimpanzee reared by his mother. Animal Cognition, 6, 259 267.

Souza, C. B. A., Dahás, L. J. S., \& Barros, R. S. (no prelo). Discriminação simples e pareamento ao modelo por identidade com estímulos bidimensionais em macacos prego infantes. Revista Brasileira de Análise do Comportamento, 7, 2. 2011..

Souza, C. B. A., Ramos, C. C., Galvão, O. F., \& Barros, R. S. (2008). Efeito do pareamento de estímulos auditivos e reforços específicos sobre a discriminação auditivo visual em macaco prego. Temas em Psicologia, 16, 199 214.

Tavares, M. C. H., \& Tomaz, C. (2002). Working memory in capuchin monkeys (Cebus apella). Behavioral Brain Research, 131, 131 137.

Tomasello, M., \& Call, J. (1997). Primate cognition. New York: Oxford University Press.

Wilkinson, K. M., \& McIlvane, W. J. (1997). Blank comparison analysis of emergent symbolic mapping by young children. Journal of Experimental Child Psychology, 67, 115 130. 